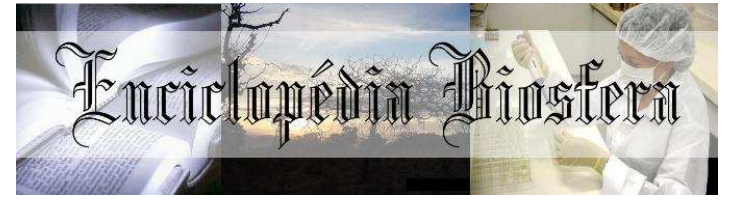

\title{
BIOMETRIA DE FRUTOS E SEMENTES DE Ceiba speciosa
}

\begin{abstract}
Soraya Silva Afonso ${ }^{1}$, Eliane Cristina Moreno de Pedri ${ }^{2^{*}}$, Vinicius Delgado da Rocha $^{3}$, Iderson Carlos Biazon ${ }^{1}$ Ana Aparecida Bandini Rossi ${ }^{4}$

${ }^{1}$ Licenciados em Ciências Biológicas pela Universidade do Estado de Mato Grosso UNEMAT, Alta Floresta, MT, Brasil.

${ }^{2}$ Mestranda do Programa de Pós Graduação em Biodiversidade e Agroecossistemas Amazônicos da Universidade do Estado de Mato Grosso - UNEMAT, Alta Floresta, MT, Brasil. *elicmbio@gmail.com

${ }^{3}$ Graduando em Bacharelado e Licenciatura em Ciências Biológicas, Universidade do Estado de Mato Grosso - UNEMAT, Alta Floresta, MT, Brasil

${ }^{4}$ Professora Doutora da Faculdade de Ciências Biológicas e Agrárias, Universidade do Estado do Mato Grosso - UNEMAT, Campus de Alta Floresta, MT, Brasil.

Recebido em: 02/10/2017 - Aprovado em: 21/11/2017 - Publicado em: 05/12/2017 DOI: 10.18677/EnciBio_2017B73

Ceiba speciosa (Malvaceae) é uma espécie arbórea nativa do Brasil, conhecida popularmente como paineira, apresenta importância econômica e ecológica. Objetivou-se neste estudo caracterizar biometricamente os frutos e sementes de $C$. speciosa ocorrentes no município de Alta Floresta, Mato Grosso. Foram amostrados 25 frutos provenientes de árvores distribuídas em fragmentos florestais. $O$ comprimento do fruto variou de 22,30 a $35,00 \mathrm{~cm}$, a largura de 63,50 a $97,27 \mathrm{~cm}$, a espessura de 65,79 a $93,28 \mathrm{~cm}$ e massa de 287,15 a 958,85 g. O número médio de sementes por fruto foi de 141,72. Em relação ás sementes, o comprimento variou de 6,17 a $8,82 \mathrm{~cm}$, a largura de 6,32 a $8,37 \mathrm{~cm}$, a espessura de 5,82 a 8,37 cm e a massa de 35,00 a 121,35 g. O valor médio de massa de paina foi 39,78 g. Ceiba speciosa apresentou maior variabilidade quanto ás características massa do fruto, número de sementes por fruto, massa da paina e massa das sementes. Verificou-se correlação significativa entre a largura e a espessura do fruto; entre a massa e o comprimento e largura do fruto, e entre a massa da paina e massa das sementes, evidenciando forte associação entre as mesmas.
\end{abstract}

PALAVRAS-CHAVE: conservação, Malvaceae, paineira.

\section{BIOMETRY OF FRUITS AND SEEDS OF Ceiba speciosa}

\begin{abstract}
Ceiba speciosa (Malvaceae) is a native tree from Brazil. It is popularly known as Paineira, presents economic and ecological importance. The objective of this study was to characterize the fruits and seeds of $C$. speciosa in the municipality of Alta Floresta, Mato Grosso, Brazil. We sampled 25 fruits from trees distributed in forest fragments. The fruits presented length from 22.30 to $35.00 \mathrm{~cm}$, width from 63.50 to
\end{abstract}


$97.27 \mathrm{~cm}$, thickness from 65.79 to $93.28 \mathrm{~cm}$ and mass from 287.15 to $958.85 \mathrm{~g}$. The average number of seeds per fruit was 141.72. The seeds showed length from 6.17 to $8.82 \mathrm{~cm}$, width from 6.32 to $8.37 \mathrm{~cm}$, thickness from 5.82 to $8.37 \mathrm{~cm}$ and mass from 35.00 to $121.35 \mathrm{~g}$. The average paina mass was $39.78 \mathrm{~g}$. Ceiba speciosa presented greater variability for characteristics: fruit mass, number of seeds per fruit, paina mass and seeds mass. There was significant correlation for width and the thickness fruit; mass, length and fruit width; paina mass and seed mass. KEYWORDS: conservation, Malvaceae, paineira.

\section{INTRODUÇÃO}

A Ceiba speciosa (A. St.-Hil.) Ravenna é uma espécie arbórea nativa do Brasil, pertencente á família Malvaceae, conhecida popularmente como paineira, paineira barriguda, paineira-rosa ou árvore-de-paina. Sua distribuição geográfica abrange os estados do Pará, Bahia, Mato Grosso, Mato Grosso do Sul, além dos estados da região sul e sudeste do Brasil (FLORA DO BRASIL, 2017).

A espécie apresenta fecundação cruzada, com reprodução mista e predominância de alogamia (SOUZA et al., 2003). As flores são hermafroditas e seus principais polinizadores são beija-flores, borboletas e morcegos; os frutos são cápsulas loculicidas, com cinco lóculos deiscentes; as sementes são oleaginosas, com presença de pêlos branco-amarelados (paina), que auxiliam a dispersão pelo vento (CARVALHO, 2003).

A paineira apresenta importância econômica e ecológica, pois pelo porte avantajado e beleza de suas flores a mesma exibe potencial para projetos paisagísticos e recuperação de áreas degradadas, bem como atua no fornecimento de alimento a fauna; a madeira por ser leve e de textura grossa é empregada na confecção de canoa, cocho, gamela e na fabricação de pasta celulósica e a paina foi muito utilizada no enchimento de almofadas e travesseiros (LORENZI, 2000; FELIX et al., 2017).

Com a destruição de habitats e a exploração inadequada dos recursos naturais tem sido observada a perda da diversidade genética de populações de árvores nativas. A variação genética dentro de uma espécie é fundamental para assegurar seu potencial adaptativo frente ás mudanças ambientais e pressões antrópicas (BORGES et al., 2016). Estudos de caracterização e a descrição biométrica de frutos e sementes podem ser utilizados na avaliação da variabilidade genética dentro e entre populações, subsidiando programas de conservação e a exploração racional dos recursos vegetais com valor econômico (BARROSO et al., 2016).

Neste contexto, o objetivo deste estudo foi caracterizar biometricamente frutos e sementes de Ceiba speciosa ocorrentes no município de Alta Floresta, Mato Grosso, visando verificar a existência de variabilidade genética intraespecífica.

\section{MATERIAL E MÉTODOS}

Foram amostrados indivíduos de $C$. speciosa distribuídos em fragmentos florestais no município de Alta Floresta, Mato Grosso. O município localiza-se na região Norte do Estado de Mato Grosso, possui uma área de $8.976,309 \mathrm{~km}^{2}$ (IBGE, 
2017) com temperatura média anual de $19,6{ }^{\circ} \mathrm{C}$ a $32,4{ }^{\circ} \mathrm{C}$ (TARIFA, 2011). A vegetação predominante é Floresta Ombrófila aberta tropical (CAIONI et al., 2014).

A coleta foi realizada no mês de julho de 2008, onde foram coletados frutos na fase final de maturação, com auxílio de um podão. Foram coletados 25 frutos de cinco indivíduos de $C$. speciosa. Os frutos foram identificados, acondicionados em embalagens de papel ainda em campo e transportados para o Laboratório de Ensino do Campus Universitário de Alta Floresta, Mato Grosso.

Para a caracterização biométrica dos frutos foram mensuradas as seguintes características: comprimento (CFR) (distância entre a base e o ápice), largura (LFR) e espessura do fruto (EFR), espessura do pericarpo (EPE) e massa do fruto fresco (MFF). Após a caracterização, os frutos foram seccionados e as sementes retiradas e lavadas em água corrente, sendo que para isolá-las do arilo foram realizadas fricções manuais em peneira de malha plástica. Em seguida avaliaram-se as seguintes características: número de sementes por fruto (NSF), comprimento (CSE), largura (LSE) e a espessura da semente (ESE), massa da paina (MPA) e das sementes (MSE). Para todas as medições utilizou-se um paquímetro digital Mitotoyo com precisão de $0,01 \mathrm{~mm}$ e/ou fita métrica e para a pesagem uma balança de precisão de 0,00001g.

A estatística descritiva, a distribuição de frequência das medidas e 0 coeficiente de correlação de Spearman foram calculados no programa BIOESTAT 5.3 (AYRES et al., 2007).

\section{RESULTADOS E DISCUSSÃO}

A análise descritiva para as variáveis analisadas nos frutos de $C$. speciosa encontra-se na Tabela 1. Observa-se variação entre os valores mínimos e máximos para todas as características avaliadas, obtendo média de $26,86 \mathrm{~cm}$ de comprimento e $79,55 \mathrm{~cm}$ de largura para o fruto. Roveri Neto e Paula (2017) e Marzinek e Mourão (2003) em estudo com C. speciosa obtiveram médias menores do que a encontrada neste estudo para o comprimento do fruto $(12,99 \mathrm{~cm}$ e $14,68 \mathrm{~cm}$, respectivamente). Essa diferença pode estar relacionada a diversos fatores, entre eles, condições ambientais e origem genética do material estudado (MOURA et al., 2010).

Para espessura do fruto, os valores variaram de 65,79 a 93,28 cm, com média de $79,70 \mathrm{~cm}$. Já a espessura do pericarpo variou de 15,19 a 23,52 cm com média de $19,24 \mathrm{~cm}$. A massa do fruto e o número de sementes oscilaram de 287,15 a 958,85 g e 65,00 a 218,00 unidades, respectivamente (Tabela 1).

TABELA 1. Estatística descritiva do comprimento (CFR), largura (LFR), espessura do fruto (EFR), espessura do pericarpo (EPE), massa dos frutos frescos (MFF) e número de sementes por fruto (NSF) de Ceiba speciosa.

\begin{tabular}{ccccccc}
\hline & CFR (cm) & LFR (cm) & EFR (cm) & EPE (cm) & MFF (g) & NSF \\
\hline Mínimo & 22,30 & 63,50 & 65,79 & 15,19 & 287,15 & 65,00 \\
Máximo & 35,00 & 97,27 & 93,28 & 23,52 & 958,85 & 218,00 \\
Média & 26,86 & 79,55 & 79,40 & 19,24 & 524,99 & 141,72 \\
Variância & 11,20 & 67,74 & 60,22 & 4,12 & 24131,45 & 1758,13 \\
Desvio Padrão & 3,35 & 8,23 & 7,76 & 2,03 & 155,34 & 41,93 \\
\hline CV (\%) & $\mathbf{1 2 , 4 6}$ & $\mathbf{1 0 , 3 5}$ & $\mathbf{9 , 8 8}$ & $\mathbf{1 0 , 5 5}$ & $\mathbf{2 9 , 5 9}$ & $\mathbf{2 9 , 5 9}$ \\
\hline CV: Coeficiente de variação & & & & &
\end{tabular}


Na Figura 1 ( $A, B, C, D, E$ e $F)$, pode ser observada a distribuição de frequência das caracteristicas biométricas dos frutos de $C$. speciosa, onde constatou-se que cerca de $32 \%$ dos frutos avaliados apresentou de 22,30 a 24,84 $\mathrm{cm}$ de comprimento. A largura dos frutos distribui-se principalmente em duas classes, que representaram $64 \%$ dos frutos amostrados, concentrando na classe de 70,25 a $77,0 \mathrm{~cm}$ cerca de $32 \%$ e na classe de 83,76 a $90,52 \mathrm{~cm}$ cerca de $32 \%$ do total dos frutos. A variável espessura do fruto, também se concentrou em duas classes $(65,79$ a $71,29 \mathrm{~cm}$ e 82,28 a $87,78 \mathrm{~cm})$ que juntas representam $48 \%$ da frequência observada.

Para distribuição de frequência da espessura do pericarpo, observou-se que $36 \%$ frutos apresentaram valores de 18,52 a $20,19 \mathrm{~cm}$. A maioria dos frutos $(44 \%)$ apresentou massa de 421,49 a 555,83g. Quanto ao número de sementes foram encontradas duas classes, que juntas representaram $48 \%$, sendo elas, 126,60 a 157,40 sementes $(24 \%)$ e 157,40 a 188,20 sementes $(24 \%)$.
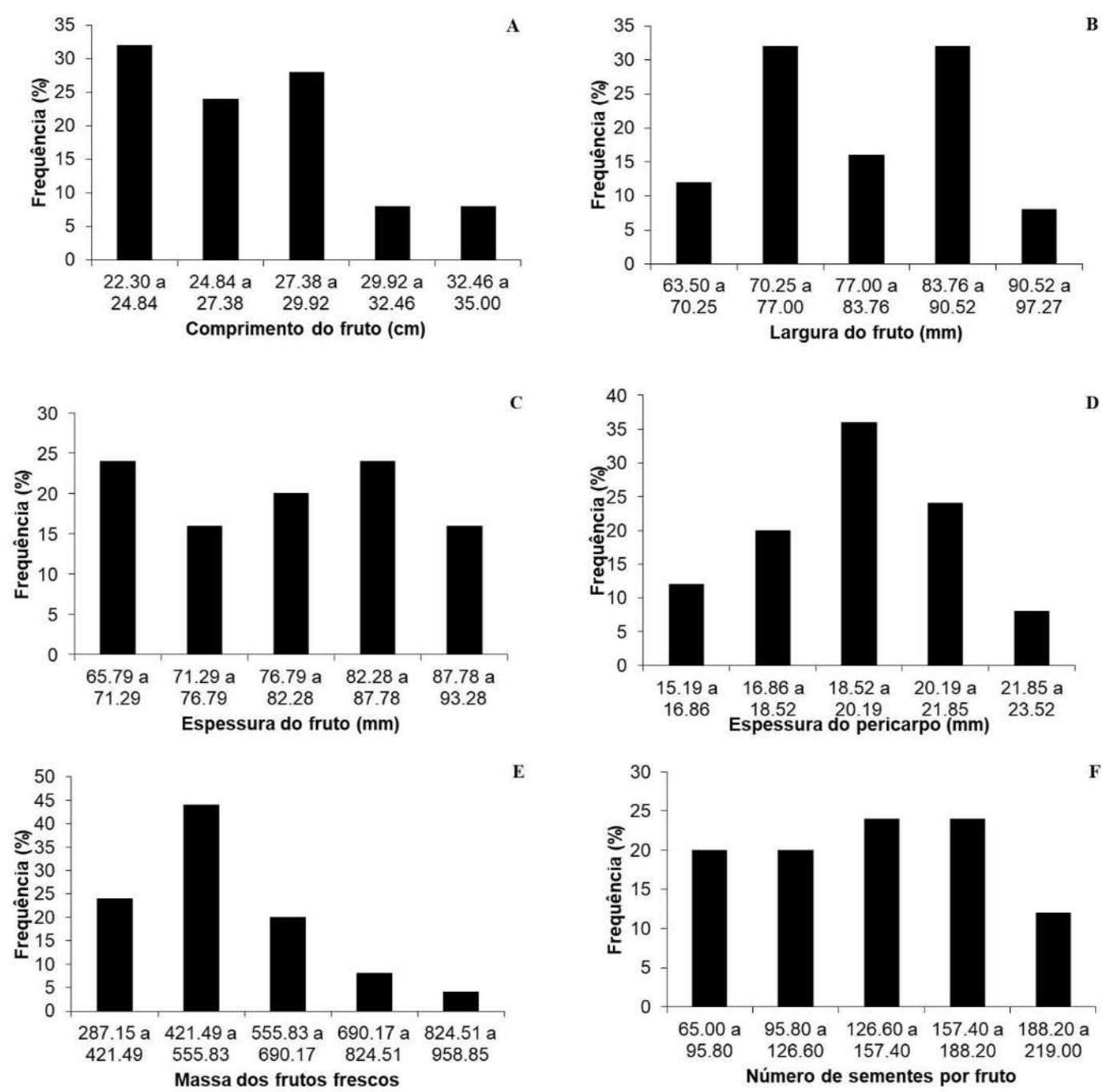

FIGURA 1. Frequência (\%) de comprimento $(A)$, largura $(B)$ e espessura do fruto $(C)$, espessura do pericarpo (D), massa dos frutos frescos $(E)$ e número de sementes por fruto de Ceiba speciosa. 
Com relação a biometria das sementes, observa-se na Tabela 2 que 0 comprimento e a largura destas, variaram de 6,17 a $8,82 \mathrm{~mm}$ e 6,32 a $8,37 \mathrm{~mm}$, respectivamente; obtendo uma média de $7,64 \mathrm{~mm}$ de comprimento e 7,61 $\mathrm{mm}$ de largura (Figura 2). Roveri Neto e Paula (2017) obtiveram menor média (5,79 mm) para o comprimento da semente. Batista et al. (2011), destacam que a variação no comprimento e massa das sementes pode ser influenciada pelos estágios de maturação dos frutos, fatores genéticos e condições climáticas sob as quais se desenvolve a planta.

$\mathrm{Na}$ variável espessura da semente, o valor médio foi $7,13 \mathrm{~cm}$, com variação de 5,82 a 8,37 cm. A massa da paina apresentou limite mínimo de 15,00 g e limite máximo de $74,45 \mathrm{~g}$, com média de $39,78 \mathrm{~g}$. Para a massa das sementes, os valores variaram de $35 \mathrm{~g}$ a 121,35 g, tendo em média $73.46 \mathrm{~g}$.

TABELA 2. Estatística descritiva do comprimento (CSE), largura (LSE) e espessura (ESE) da semente, massa da paina (MPA) e massa das sementes (MSE) de Ceiba speciosa.

\begin{tabular}{cccccc}
\hline $\begin{array}{c}\text { Medidas } \\
\text { Estatísticas }\end{array}$ & CSE (mm) & LSE (mm) & ESE (mm) & MPA (g) & MSE (g) \\
\hline Mínimo & 6,17 & 6,32 & 5,82 & 15,00 & 35,00 \\
Máximo & 8,82 & 8,37 & 8,37 & 74,45 & 121,35 \\
Média & 7,64 & 7,61 & 7,13 & 39,78 & 73,46 \\
Variância & 0,30 & 0,17 & 0,34 & 204,93 & 437,64 \\
Desvio Padrão & 0,55 & 0,41 & 0,58 & 14,32 & 20,92 \\
\hline CV (\%) & $\mathbf{7 , 1 8}$ & $\mathbf{5 , 3 4}$ & $\mathbf{8 , 2 0}$ & $\mathbf{3 5 , 9 9}$ & $\mathbf{2 8 , 4 8}$ \\
\hline CV: Coeficiente de variação & & & &
\end{tabular}

A distribuição de frequência para o comprimento da semente mostra que $20 \%$ das mesmas medem de 7,23 a 7,76 mm (Figura 1A). Cerca de $48 \%$ das sementes apresentaram largura em uma classe central de 7,55 a 7,96 mm (Figura 2B). A espessura da semente concentrou-se na classe de 6,84 a $7,35 \mathrm{~mm}$, representando $36 \%$ das sementes amostradas (Figura $2 \mathrm{C}$ ). Já a massa da paina concentrou-se em duas classes principais que juntas representam $68 \%$, que foram de 26,89 a $50,67 \mathrm{~g}$ (Figura 2D). Dentre as sementes amostradas, $36 \%$ apresentaram massa na classe de 52,27 a $69,54 \mathrm{~g}$. 

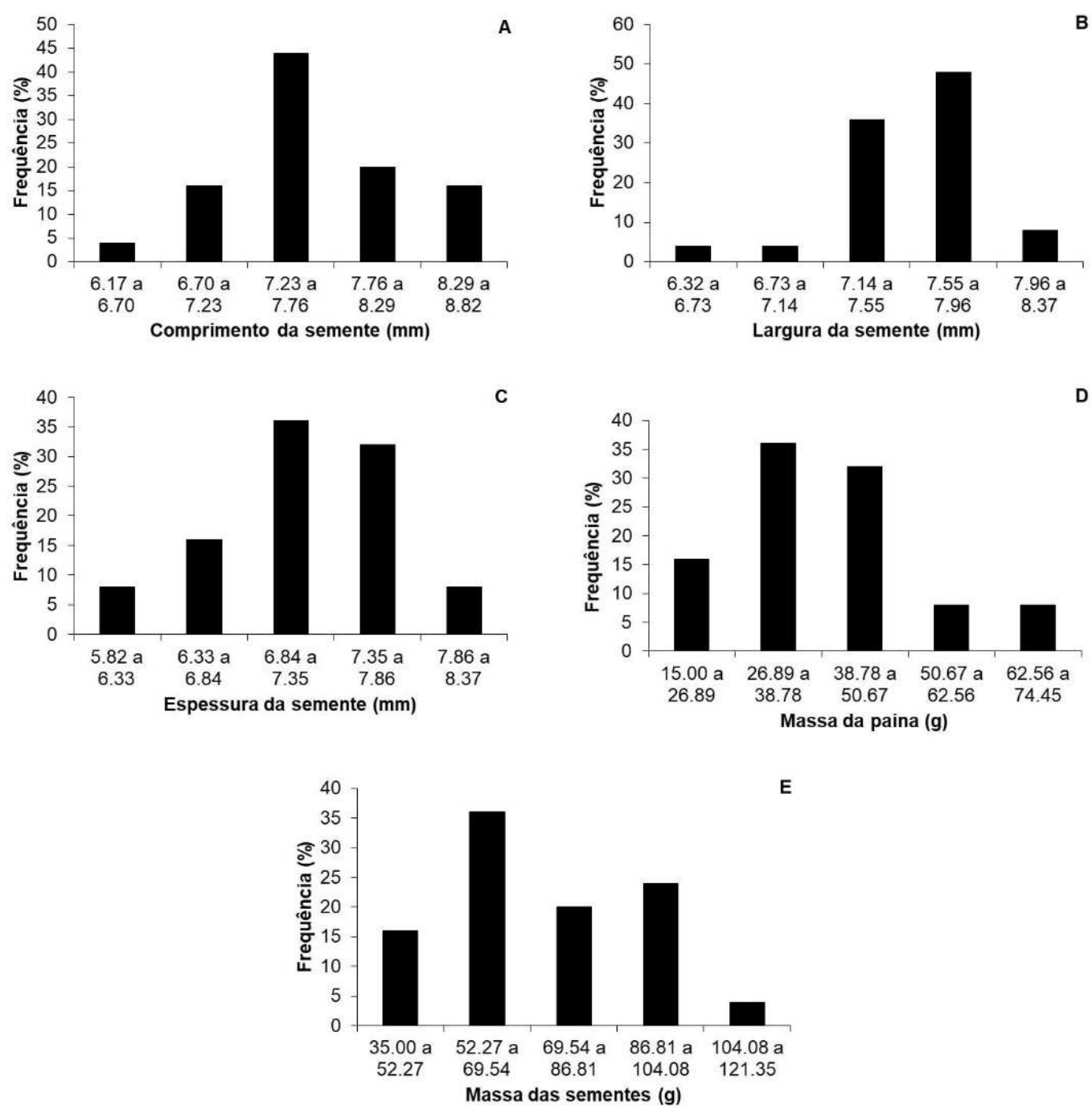

FIGURA 2. Frequência (\%) de comprimento (A), largura (B) e espessura da semente (C), massa da paina (D) e massa das sementes de Ceiba speciosa.

As estimativas dos coeficientes de correlação de Spearman (rS) entre as características biométricas de frutos e sementes de $C$. speciosa encontram-se na tabela 3 , onde observou-se que a maior estimativa de correlação ocorreu entre a largura e a espessura do fruto $(0,834)$. A variável massa do fruto (MFF) correlacionou de forma positiva e significativa com comprimento do fruto (CFR) e largura do fruto (LFR), indicando que quanto maior o tamanho do fruto (comprimento e largura) maior a massa do mesmo. Gonçalves et al. (2013) ao estudarem frutos e sementes de Hancornia speciosa Gomes ocorrentes na região de Nova Xavantina, Mato Grosso, verificaram que comprimento e largura do fruto se correlacionaram com massa do fruto, corroborando assim com correlações obtidas neste estudo.

O conhecimento da correlação entre as variáveis auxilia o processo de seleção indireta de características de interesse com difícil mensuração em campo. Para C. speciosa pode-se inferir que em campo a seleção indireta de frutos com maior massa de paina pode ser realizada a partir de frutos de maior massa fresca, 
uma vez que estas características (MFF e MPA) estão correlacionadas positivamente.

TABELA 3. Correlação entre as variáveis dos frutos e sementes de Ceiba speciosa. Comprimento (CFR), largura (LFR) e espessura (EFR) do fruto, espessura do pericarpo (EPE), massa do fruto fresco (MFF), número de sementes por fruto (NSE), comprimento (CSE), largura (LSE) e espessura (ESE) da semente, massa da paina (MPA) e massa das sementes (MSE).

\begin{tabular}{|c|c|c|c|c|c|c|c|c|c|c|c|}
\hline & CFR & LFR & EFR & EPE & MFF & NSF & CSE & LSE & ESE & MPA & MSE \\
\hline CFR & 1 & & & & & & & & & & \\
\hline LFR & $0,696^{\star *}$ & 1 & & & & & & & & & \\
\hline EFR & $0,659^{* *}$ & $0,834^{\star *}$ & 1 & & & & & & & & \\
\hline EPE & $0,505^{\star \star}$ & $0,612^{\star *}$ & $0,483^{*}$ & 1 & & & & & & & \\
\hline MFF & $0,730^{\star \star}$ & $0,804^{\star *}$ & $0,591^{\star \star}$ & $0,399^{*}$ & 1 & & & & & & \\
\hline NSF & $0,121^{\text {ns }}$ & $0,122^{\mathrm{ns}}$ & $-0,191^{\mathrm{ns}}$ & $0,221^{\mathrm{ns}}$ & $0,315^{\text {ns }}$ & 1 & & & & & \\
\hline CSE & $0,084^{\mathrm{ns}}$ & $0,231^{\mathrm{ns}}$ & $0,054^{\mathrm{ns}}$ & $-0,150^{\text {ns }}$ & $0,252^{\text {ns }}$ & $-0,014^{\mathrm{ns}}$ & 1 & & & & \\
\hline LSE & $0,002^{\text {ns }}$ & $-0,127^{\text {ns }}$ & $-0,033^{\text {ns }}$ & $-0,186^{\text {ns }}$ & $0,059^{\text {ns }}$ & $-0,223^{\text {ns }}$ & $-0,415^{*}$ & 1 & & & \\
\hline ESE & $-0,135^{\text {ns }}$ & $-0,460^{*}$ & $-0,301^{\mathrm{ns}}$ & $-0,179^{\text {ns }}$ & $-0,313^{\text {ns }}$ & $0,082^{\mathrm{ns}}$ & $0,129^{\text {ns }}$ & $-0,339^{\text {ns }}$ & 1 & & \\
\hline MPA & $0,299^{\text {ns }}$ & $0,134^{\mathrm{ns}}$ & $-0,052^{\text {ns }}$ & $-0,011^{\mathrm{ns}}$ & $0,482^{\star}$ & $0,442^{*}$ & $-0,076^{\text {ns }}$ & $0,341^{\mathrm{ns}}$ & $-0,257^{\text {ns }}$ & 1 & \\
\hline MSE & $0,493^{*}$ & $0,260^{\text {ns }}$ & $0,106^{\mathrm{ns}}$ & $0,144^{\mathrm{ns}}$ & $0,498^{*}$ & $0,521^{* *}$ & $-0,037^{\text {ns }}$ & $0,153^{\text {ns }}$ & $-0,130^{\text {ns }}$ & $0,788^{\star *}$ & 1 \\
\hline
\end{tabular}

**significativo a $1 \%$ de probabilidade; *significativo a $5 \%$ de probabilidade; ns: não significativo.

Neste estudo constatou-se grande variação entre os valores máximo e mínimo de massa do fruto (MFF), número de sementes por fruto (NSF), massa da paina (MPPA) e massa das sementes (MSE). Esses resultados revelam uma alta heterogeneidade nestes parâmetros avaliados, evidenciando a existência de variação entre as amostras coletadas. A variação encontrada nos frutos e sementes de C. speciosa pode ser promovida por componentes ambientais ou pela variabilidade genética dos indivíduos, como já obervado em outras espécies nativas: Mauritia flexuosa L.f (MATOS et al., 2014) e Bertholletia excelsa Bonpl. (ROCHA et al., 2016). De acordo com Araújo et al. (2015), em espécies nativas há uma maior variabilidade quanto ás características morfológicas de frutos e sementes, o que pode ser explicada pela alta diversidade genética existente entre as plantas.

\section{CONCLUSÃO}

A espécie Ceiba speciosa apresentou maior variabilidade em relação às características massa do fruto, número de sementes por fruto, massa da paina e massa das sementes. Houve correlação positiva e significativa entre a largura e a espessura do fruto; entre a massa e o comprimento e largura do fruto e entre a massa da paina e massa das sementes. A seleção de frutos com maior massa de paina pode ser realizada por meio da massa fresca do fruto.

\section{AGRADECIMENTOS}

À Coordenação de Aperfeiçoamento de Pessoal de Nível Superior (CAPES) pela concessão de bolsa de mestrado ao segundo autor, ao Conselho Nacional de 
Desenvolvimento Científico e Tecnológico (CNPq) pela concessão de bolsa de Iniciação Cientifica ao terceiro autor e à Universidade do Estado de Mato Grosso (UNEMAT), Campus de Alta Floresta, pela oportunidade de estudo bem como a disposição dos profissionais.

\section{REFERÊNCIAS}

ARAÚJO, B. A.; SILVA, M. C. B.; MOREIRA, F. J. C.; SILVA, K. F.; TAVARES, M. K. N. Caracterização biométrica de frutos e sementes, química e rendimento de polpa de juazeiro (Ziziphus joaseiro Mart.). Agropecuária Científica no Semi-Árido, v. 11, n. $2, \quad$ p. $15-21, \quad 2015 . \quad$ Disponível em: $<$ http://revistas.ufcg.edu.br/acsa/index.php/ACSA/article/view/605/pdf>.

AYRES, M.; AYRES JUNIOR, M.; AYRES, D.L.; SANTOS, A.S. BioEstat: Aplicações estatísticas nas áreas das ciências bio-médicas. Belém: Sociedade Civil Mamirauá, 2007.

BARROSO, R. F.; SILVA, F. A. NÓBREGA, J. S.; SILVA E SILVA, L. J.; NOVAIS, D. B.; FERREIRA, V. S. Biometria de frutos e sementes de Luetzelburgia auriculata (Allemão) Ducke. Revista Verde de Agroecologia e Desenvolvimento Sustentável, v. $11, \quad$ n. $5, \quad$ p. 155-160, 2016. Disponível em: <http://www.gvaa.com.br/revista/index.php/RVADS/article/view/4703/4241>. DOI: 10.18378/rvads.v11i5.4703.

BATISTA, G.S.; COSTA, R.S.; GIMENES, R.; PIVETTA, K.F.L.; MÔRO, F.V. Aspectos morfológicos dos diásporos e das plântulas de Syagrus oleracea (Mart) Becc - Arecaceae. Comunicata Scientiae, v. 2, n. 3, p. 170-176, 2011. Disponível em: <https://dialnet.unirioja.es/descarga/articulo/3985477>.

BORGES, R. C.; SANTOS, F. M. G.; MAIA, M. C. C.; LIMA, P. S. C.; VALENTE, S. E. S. Investigating genetic diversity in sapucaia using inter simple sequence repeat markers. Genetics and Molecular Research, v. 15, n. 3, p. 1-13, 2016. Disponível em: <http://www.funpecrp.com.br/gmr/year2016/vol15-3/pdf/gmr8565.pdf>. DOI: $10.4238 / g m r .15038565$.

CAIONI, C.; CAIONI; S.; PARENTE, T.L.; SILVEIRO, A.C.; CLAUDINO, W.V.;. Dinâmica da temperatura superficial no perímetro urbano de Alta Floresta/MT. Enciclopédia Biosfera, v. 10, n. 18, 2014. Disponível em: <http://www.conhecer.org.br/enciclop/2014a/MULTIDISCIPLINAR/dinamica.pdf>

CARVALHO, P. E. R. Espécies arbóreas brasileiras. Brasília: Embrapa Informação Tecnológica, 2003.

FELIX, F. C.; OLIVEIRA, D. F.; QUERIOZ, J. P. C. G.; ARAÚJO, F. S.; PACHECO, M. V. Qualidade fisiológica de sementes de Ceiba speciosa em relação a níveis de umedecimento e substrato. Tecnologia e Ciência Agropecuária, v. 11, n. 3, p. 7580, 2017. Disponível em: <http://revistatca.pb.gov.br/edicoes/volume-11- 
2017/copy_of_v-11-n-1-marco-2017/germinacao-de-sementes-de-ceibaspeciosa.pdf>.

FLORA DO BRASIL. Rubiaceae. In: Flora do Brasil 2020 em construção. Jardim Botânico do Rio de Janeiro, 2017. Disponível em: <http://floradobrasil.jbrj.gov.br/reflora/floradobrasil/FB87533>. Acesso em: 04 Ago. de 2017.

GONÇALVES, L. G. V.; ANDRADE, F. R.; MARIMON JUNIOR, B. H.; SCHOSSLER, T. R.; LENZA, E.; MARIMON, B. S. Biometria de frutos e sementes de mangaba (Hancornia speciosa Gomes) em vegetação natural na região leste de Mato Grosso, Brasil. Revista de Ciências Agrárias, v. 36, n.1, p. 31-40, 2013. Disponível em: <http://www.scielo.mec.pt/scielo.php?script=sci_arttext\&pid=S0871-

018X2013000100006>.

IBGE - Instituto Brasileiro de Geografia e Estatística. Geociência - Área Territorial Oficial.

https://cidades.ibge.gov.br/v4/brasil/mt/altafloresta/panorama. Acesso em: 05 set 2017.

LORENZI, H. Árvores brasileiras: manual de identificação e cultivo de plantas arbóreas nativas do Brasil. 3.ed. Nova Odessa: Instituto Plantarum, 2000.

MARZINEK, J.; MOURAO, K. S. M. Morphology and anatomy of the fruit and seed in development of Chorisia speciosa A, St,-Hil, - Bombacaceae. Revista Brasileira de Botânica, v. 26, n. 1, 2003. Disponível em: <http://www.scielo.br/pdf/rbb/v26n1/v26n1a04>.

MATOS, F. S.; NUNES, Y. R. F.; SILVA, M. A. P.; OLIVEIRA, I. S. Variação biométrica de Diásporos de Buriti (Mauritia flexuosa Lf-Arecaceae) em veredas em diferentes estágios de conservação. Ciência Florestal, v. 24, n. 4, p.833-842, 2014. Disponível em: <http://www.scielo.br/pdf/cflo/v24n4/0103-9954-cflo-24-04-00833>.

MOURA, R. C.; LOPES, P. S. N.; BRANDÃO JUNIOR, D. S.; GOMES, J. G.; PEREIRA, M. B. Biometria de frutos e sementes de Butia capitata (Mart.) Beccari (Arecaceae), em vegetação natural no Norte de Minas Gerais, Brasil. Biota Neotropica, v. 10, n. 2. p. 415-419, 2010. Disponível em: <http://www.scielo.br/pdf/bn/v10n2/40.pdf. $\quad$ DOI: 10.1590/S167606032010000200040>.

ROCHA, V. D.; LIMA, J. S.; BISPO, R. B.; COCHEV, J. S.; ROSSI, A. A. B. Caracterização biométrica de frutos e sementes de Castanha-do-Brasil na Amazônia Mato-Grossense. Enciclopédia Biosfera, v. 13, n. 29, p. 185-195, 2016. Disponível em: <www.conhecer.org.br/enciclop/2016b/agrarias/caracterizacao\%20biometrica>. DOI: 10.18677/EnciBio_2016B_017. 
ROVERI NETO, A.; PAULA, R. C. Variabilidade entre árvores matrizes de Ceiba speciosa St. Hil para caracteristicas de frutos e sementes. Revista Ciência Agronômica, v. 48, n. 2, p. 318-327, 2017. DOI: 10.5935/1806-6690.20170037. Disponível em: < www.scielo.br/pdf/rca/v48n2/1806-6690-rca-48-02-0318>.

SOUZA, L. M. F. I.; KAGEYAMA, P. Y.; SEBBENN, A. M. Sistema de reprodução em população natural de Chorisia speciosa A. St.-Hil. (Bombacaceae). Revista Brasileira de Botânica, v. 26, n. 1, p. 113-121, 2003. Disponível em: $<$ <ttp://www.scielo.br/pdf/rbb/v26n1/v26n1a12>.

TARIFA, J.R. Mato Grosso: clima - análise e representação cartográfica. Cuiabá: Entrelinhas, 2011. 